\title{
Vitrectomy technique in anterior segment surgery
}

\author{
J. J. KANSKI AND M. D. P. CRICK \\ From Moorfields Eye Hospital, High Holborn, London, WCI
}

SUMMARY Instruments designed primarily for vitreous surgery were used in 74 anterior segment operations. In each case the instrument was introduced into the eye through a limbal incision. This preliminary report shows that this new approach offers several advantages over conventional methods in selected cases. It is of particular value in the management of presenile and complicated cataracts and in the removal of capsulo-lenticular remnants and pupillary membranes. A larger number of cases and longer follow-up are necessary to define further the specific indications and limitations of this technique.

The recent development of instruments that will cut and remove ocular tissue through a very small incision represents a significant departure from conventional ophthalmic surgical procedures. Most instruments consist of 2 metallic tubes (Machemer et al., 1971; Peyman and Dodich, 1971; O'Malley and Heintz, 1972; Douvas, 1973; Kloti, 1973; Federman et al., 1974). Tissue aspirated into the tip of the outer tube is cut by a shearing action produced by the rotation or oscillation of the inner tube. Evacuation is performed by suction from an aspirating syringe that draws the tissue into the inlet and removes it from the eye. The inflow of fluid is controlled by gravity. The instrument can be introduced into the eye through an incision at the limbus or pars plana.

The purpose of this report is to present a preliminary analysis of our experience in anterior segment surgery using instruments containing infusion, aspiration, and cutting features as designed for vitreous surgery.

\section{Patients and methods}

During 13-months' period (January 1976 to January 1977), 74 anterior segment operations were performed with a vitrectomy instrument; 53 were performed with the Kloti vitreous stripper and 21 with the Keeler-Federman SITE. The patients (31 females and 60 males) ranged in age from 6 months to 63 years. 8 patients had both eyes operated on at different times, and a further patient had 2 operations on 1 eye and 1 operation on the other eye.

Ocular evaluation included best corrected visual

Address for reprints: J. J. Kanski, FRCS, Moorfields Eye Hospital, High Holborn, London, WC1 acuity, external slit-lamp biomicroscopy, applanation tonometry, and ultrasonography in selected cases. Pre- and postoperative anterior segment photographs were obtained when possible.

Patients were usually admitted to hospital 1 or 2 days before the operation and remained 4 to 7 days after it. 71 operations were performed under general anaesthetic, 2 under neuroleptic anaesthetic, and 1 under local anaesthetic. After the completion of surgery all eyes were given a subconjunctival injection of gentamicin and betamethasone. Mydriatic and antibiotic-steroid drops were instilled frequently after operation. After discharge, patients were followed up at 2- to 4-weekly intervals until a stable state had been reached.

Of the 54 eyes that underwent lensectomy 35 had cataracts due to chronic anterior uveitis; extensive posterior synechiae were present in the majority of these eyes. Of the 6 eyes with congenital cataracts 2 were thought to have been due to maternal rubella. Of the 9 eyes with traumatic cataracts 5 had suffered penetrating injuries and 4 blunt trauma. There were 3 eyes with presenile cataracts, and one 63-year-old patient with a posterior subcapsular cataract underwent lensectomy during a combined lensectomy-vitrectomy procedure.

Of the 16 eyes that had anterior segment reconstruction for the removal of capsulo-lenticular remnants, pupillary membranes, or pupil enlargement, 2 had had previous surgery for traumatic cataracts, 5 for congenital cataracts, and 9 for complicated cataracts due to chronic anterior uveitis. In 4 eyes a traumatically subluxated lens was removed.

\section{Operative technique}

A wire lid speculum is inserted and the eye is steadied 


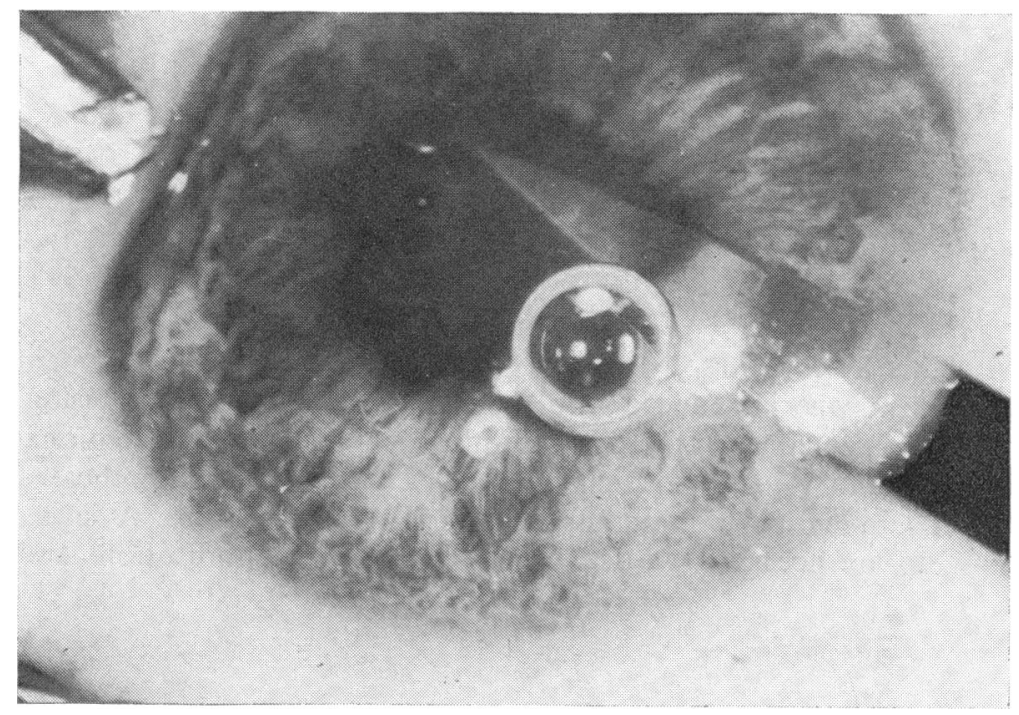

Fig. 1 Lensectomy of cataract secondary to chronic anterior. uveitis. An oblique stab incision is made with a Graefe knife at 10 o'clock just inside the limbus. The knife is then passed into the lens nucleus

with fixation forceps; traction sutures are not required. An oblique incision at 10 o'clock just inside the limbus is made with a Graefe knife (Fig. 1). The hardness of the lens nucleus is then tested. If the knife does not penetrate the nucleus easily, an alternative method of extraction has to be used. It is important to avoid damaging the posterior capsule at this stage and not to dislocate the lens by probing too forcibly at a hard nucleus. If the cataract is suitable for lensectomy the corneal incision is enlarged. An overtight incision may leak postoperatively owing to stretching of the corneal tissues, and too large an incision will leak during the operation.

After the instrument is introduced into the eye (Fig. 2) as much lens matter as possible is removed working within the capsular bag (Fig. 3). This minimises excessive irrigation of the corneal endothelium. The assistant should wait until he sees the tip of the instrument applied to the lens matter and then aspirate fairly forcibly so as to engage it into the aspiration port (Fig. 4). Sometimes the lens fibres will not aspirate immediately, but if suction is maintained for a few moments they

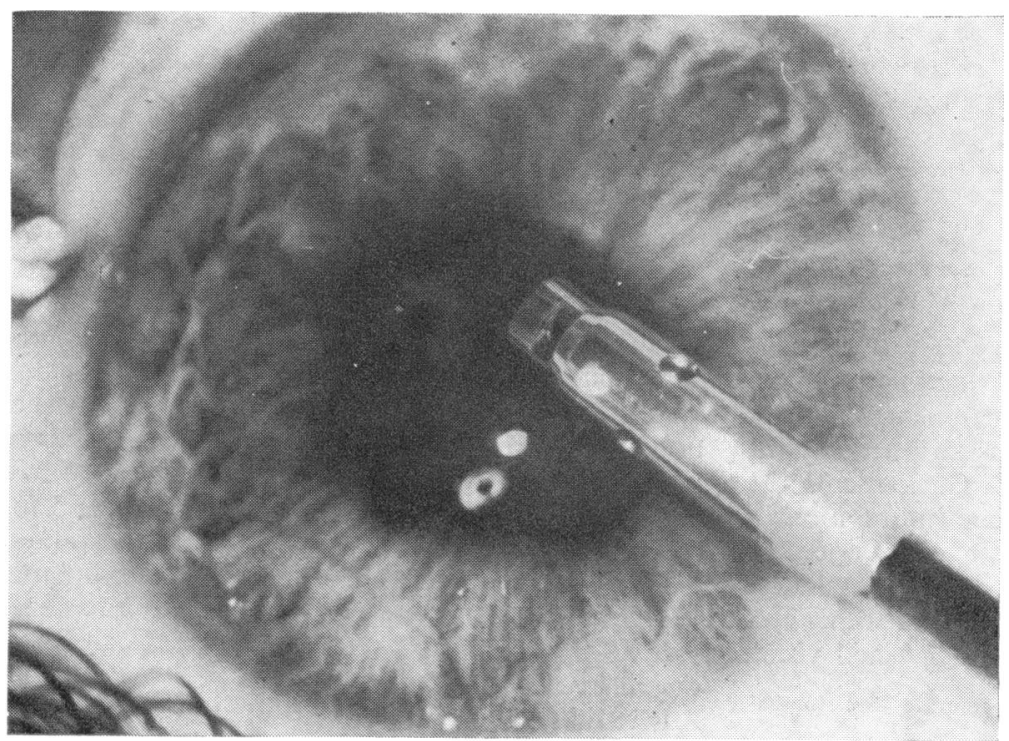

Fig. 2 The incision is slightly enlarged and the vitrectomy instrument is introduced into the anterior chamber along the track made by the knife 


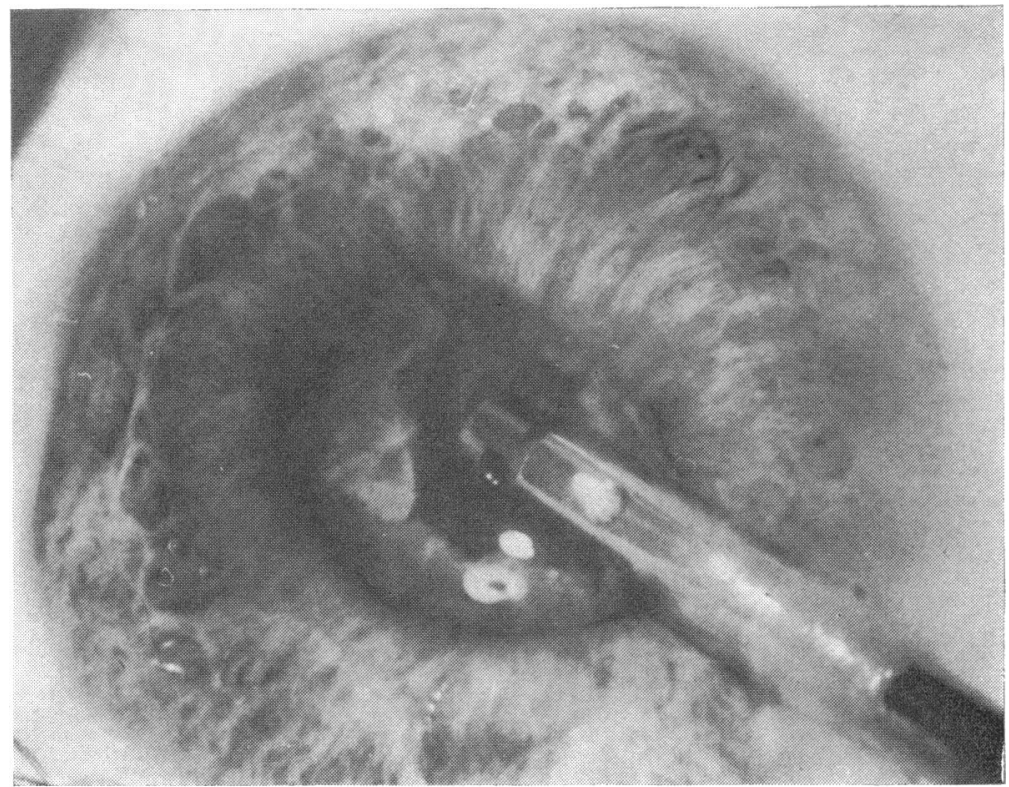

Fig. 3 Lensectomy is commenced by aspiration and clutting

deform and then aspirate quite suddenly. When this occurs the assistant should stop aspirating so as not to collapse the anterior chamber. Anterior chamber collapse can be avoided quite easily, and computer-controlled infusion and aspiration does not appear to be necessary. As much soft lens matter as possible is removed in this way without operating the cutting mechanism, though cutting will speed up the process and is necessary for harder lens material and also for capsule, cyclitic membranes, iris, and vitreous.

After as much lens matter as possible has been aspirated the anterior capsule is removed and, if necessary, the pupil enlarged. The remaining lens matter is then dislocated from within the capsular bag and drawn into the centre of the pupil or into the anterior chamber. The posterior and peripheral layers of the lens can be peeled away from the

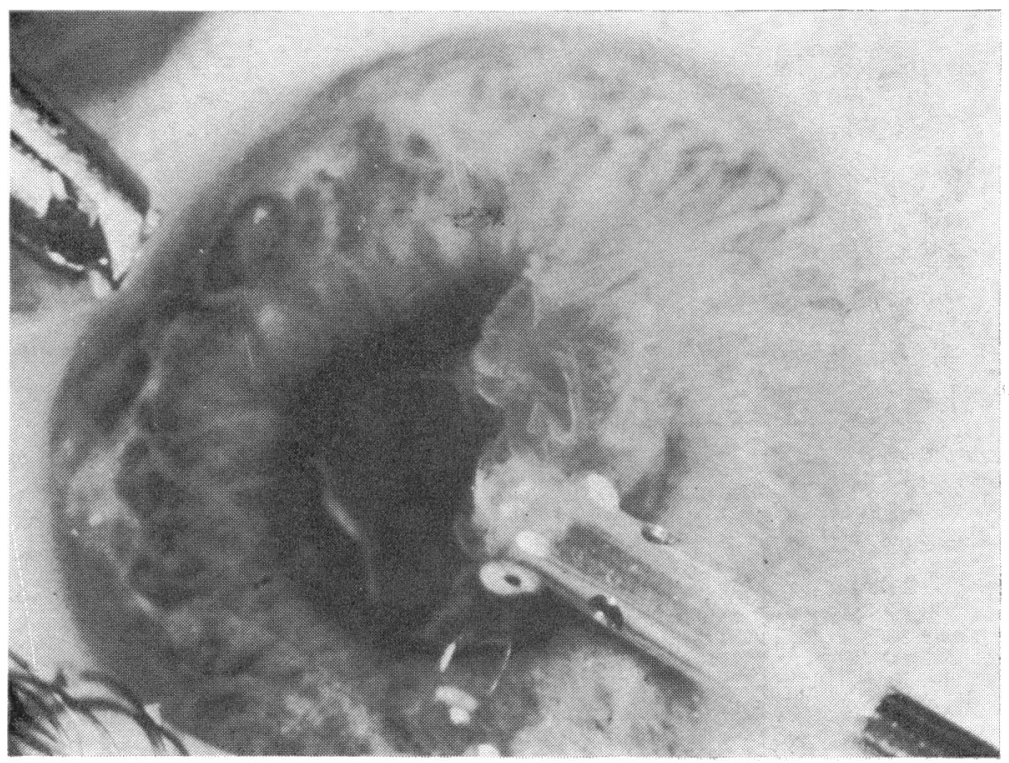

Fig. 4 Lensectomy is nearing completion. The posterior capsule is still intact in order to prevent lens material from falling into the vitreous cavity 


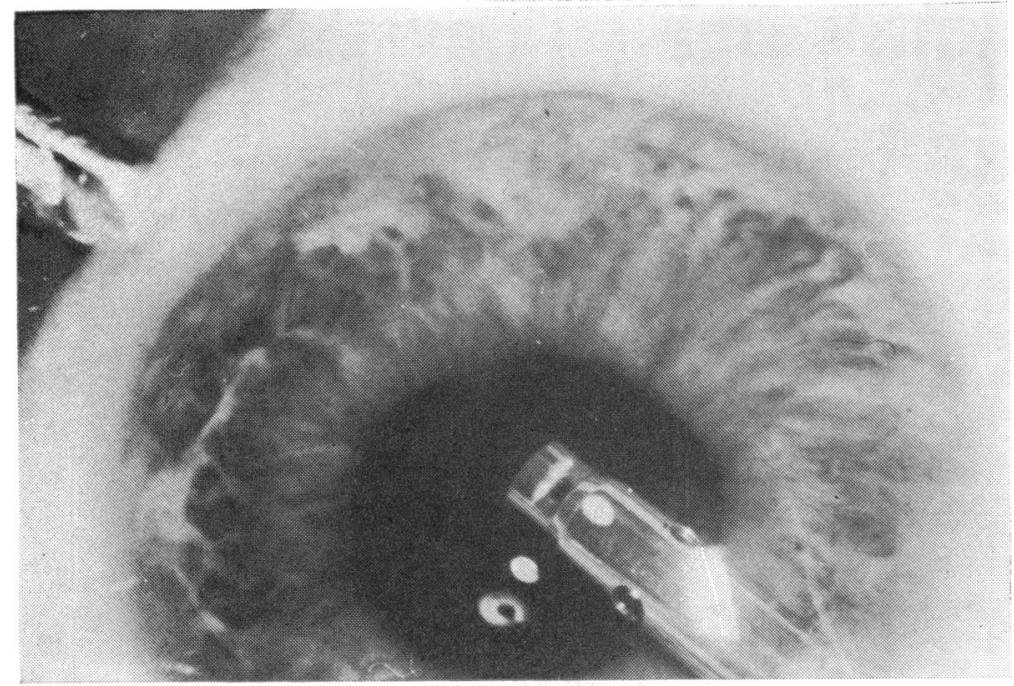

Fig. 5 Eye after completion of lensectomy and removal of posterior capsule

posterior capsule. Allowing the anterior chamber to collapse slightly at this stage helps to express lens matter from under the iris into the centre of the pupil. It is important to avoid aspirating the vitreous base so as not to cause traction and subsequent retinal dialysis. Finally, the posterior capsule is removed and a shallow anterior vitrectomy performed. It is important to remove the posterior capsule in eyes with chronic iridocyclitis so as to prevent the formation of postoperative membranes. In eyes with congenital cataracts it is possible to leave the posterior capsule behind. The instrument is then removed from the eye (Fig. 5) and air injected into the anterior chamber. The bubble should extend as far as the internal opening of the incision so as to prevent the formation of anterior synechiae. No sutures are necessary unless the incision has inadvertently been made too vertical or the air is not retained.

Bleeding during the operation from the iris or vascularised membranes usually stops spontaneously; occasionally it may be necessary to cauterise the bleeding point (Fig. 6).

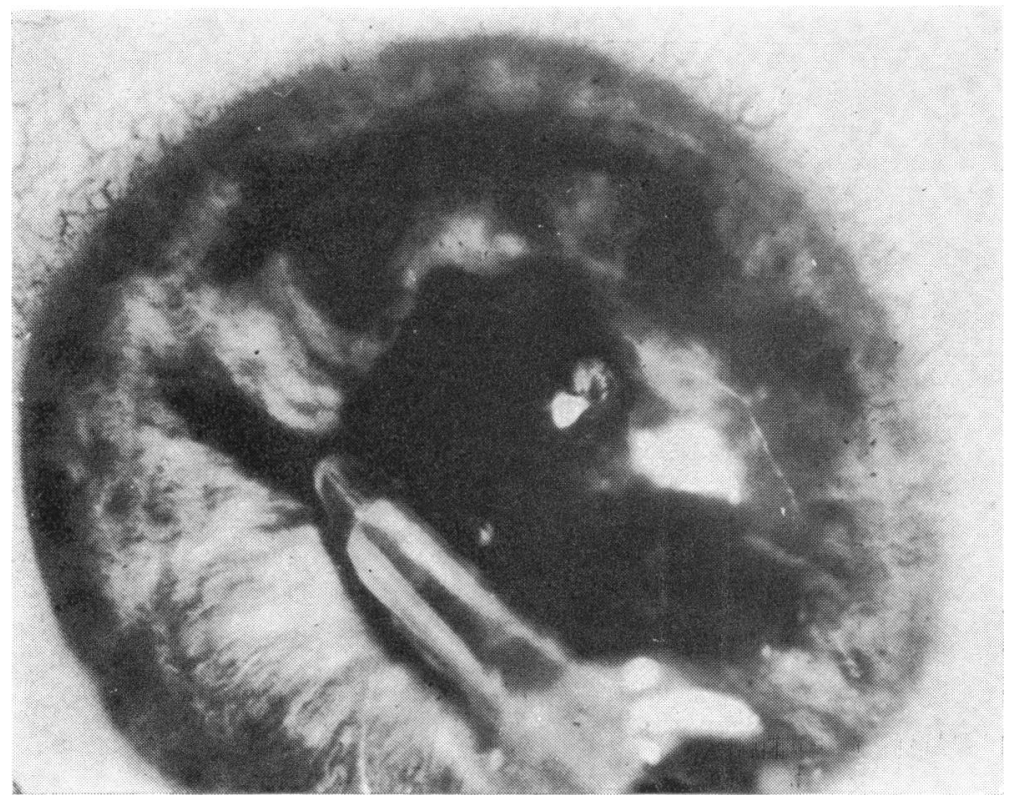

Fig. 6 Cauterisation of bleeding iris vessels 


\section{Results}

Lensectomy (54 eyes)

Accurate assessment in visual acuity was possible in 45 eyes. It can be seen from Table 1 that in 40 eyes $(89 \%)$ the preoperative visual acuity was 'counting fingers' or less, and the postoperative visual acuity of $6 / 12$ or better was achieved in 17 eyes $(38 \%)$. 36 eyes had an improvement in visual acuity, in 7 eyes there was no change owing to preexisting lesions (retinal detachment in 2 and gross field loss in 1), and 2 eyes developed retinal detachment postoperatively.

In 3 eyes the lensectomy failed as the lens was calcified. The section was enlarged and the cataract was removed with a cryoprobe. In another eye only a partial lensectomy was possible owing to unexpected hardness of the lens. Fortunately, the remaining lens material absorbed spontaneously during the next 8 weeks.

In 6 eyes there was loss of lens material into the vitreous cavity during the operation. In 4 eyes the amount of lens material was very small and in each case it absorbed spontaneously. In 2 eyes the whole nucleus was lost. An unsuccessful attempt to retrieve it was made in 1 eye with the vitrectomy instrument. This resulted in the formation of a retinal tear and subsequent inoperable retinal detachment. The nucleus was later removed from the anterior chamber with the cryoprobe. In another eye a retinal detachment formed 6 weeks after an uneventful lensectomy. It is thought that this was the result of excessive traction on the vitreous base by the vitrectomy instrument resulting in the formation of a dialysis.

Transient striate keratitis occurred in 5 eyes. In each case this followed prolonged manipulation of lens material in the anterior chamber of eyes with relatively hard nuclei. It seems probable that this resulted in endothelial damage.

Reoperation was necessary in only 1 eye. This was to remove a pupillary membrane which was the result of postoperative bleeding. A bleeding disorder

Table 1 Visual acuity in 54 eyes before and after lensectomy

\begin{tabular}{lcc}
\hline Visual acuity & No. of eyes & \\
& Preoperative & Postoperative \\
\cline { 2 - 2 } 6/12 or better & 0 & 17 \\
$6 / 18-6 / 24$ & 3 & 7 \\
$6 / 36-6 / 60$ & 2 & 7 \\
Counting fingers & 11 & 3 \\
Hand movements & 13 & 5 \\
Light perception & 16 & 6 \\
Too young to measure & 9 & 9 \\
Total & 54 & 54 \\
\hline
\end{tabular}

Table 2 Complications of lensectomy in 54 eyes

\begin{tabular}{ll}
\hline & No. of eyes \\
\hline $\begin{array}{l}\text { Surgical complications: } \\
\quad \text { Partial lens removal } \\
\text { Loss of lens nucleus into vitrous }\end{array}$ & 1 \\
Postoperative complications: & \\
$\quad$ Transient striate keratitis & \\
$\quad$ Formation of pupillary membranc & 1 \\
Retinal detachment & 2 \\
\hline
\end{tabular}

was suspected in this patient. A summary of the complications is listed in Table 2.

On the whole, eyes with chronic uveitis tolerated lensectomy very well, with no significant reactivation of uveitis during the postoperative period despite the fact that over $60 \%$ of eyes had active inflammation at the time of surgery.

\section{Anterior segment reconstruction (I6 eyes)}

Successful removal of capsulo-lenticular remnants (Fig. $7 a, b$ ), pupillary membranes, and pupillary

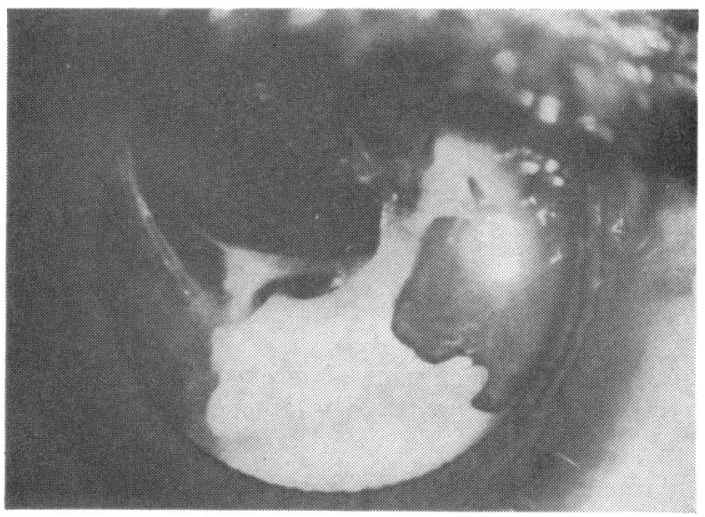

a

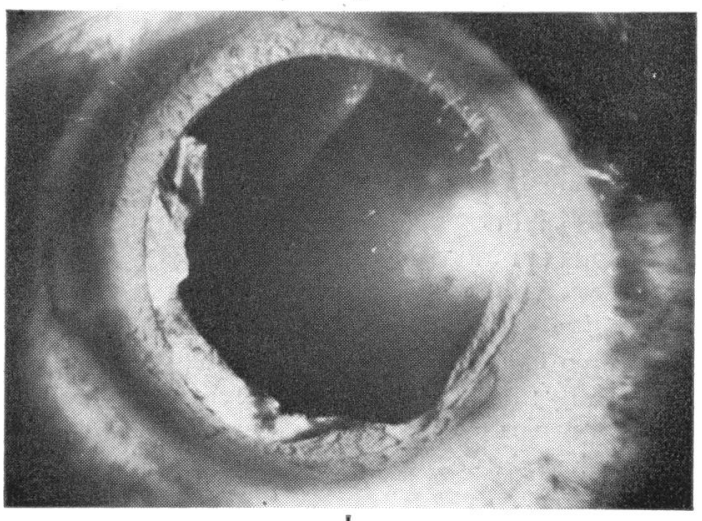

b

Fig. 7 (a) Capsulo-lenticular remnants after conventional congenital cataract surgery. (b) Appearance after operation with vitrectomy instrument 


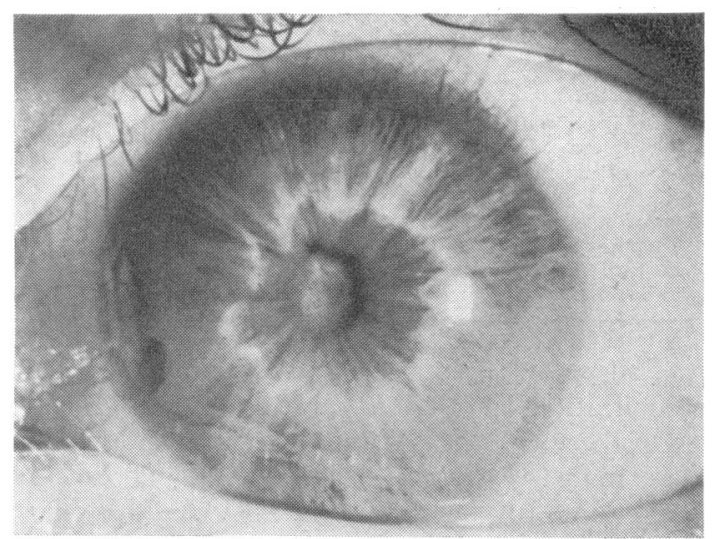

a

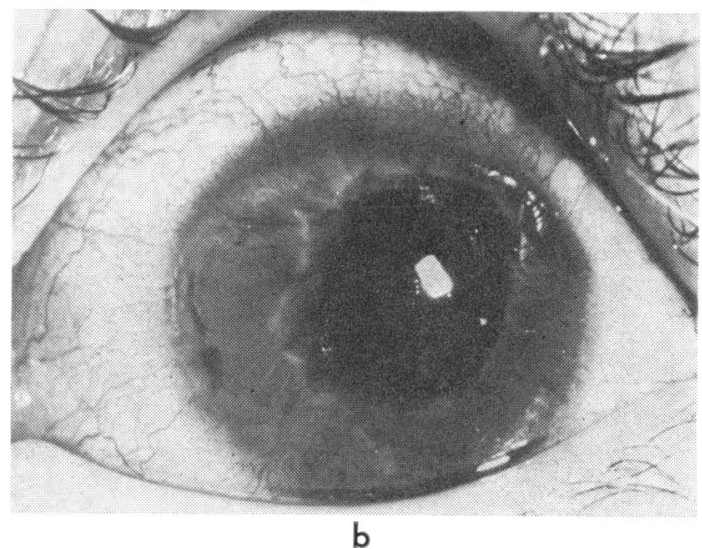

Fig. 8 (a) Preoperative appearance of cataract secondary to chronic anterior uveitis. (b) Postoperative appearance

enlargement with the vitrectomy instrument was achieved in 14 eyes. In 1 eye with a very tough posttraumatic membrane, a two-instrument technique using Vanas scissors was necessary in order to achieve a pupillary opening. In another eye with a suspected persistent hyperplastic primary vitreous it was impossible to obtain an opening.

Although in the majority of eyes visual improvement was slight owing to pre-existing retinal damage, no serious complications resulted.

\section{Removal of subluxated lens (14 eyes)}

A traumatically subluxated cataractous lens was successfully removed in 4 eyes. A preliminary anterior vitrectomy was performed, the incision was enlarged, and the lens was extracted with the cryoprobe.

\section{Discussion}

On the whole the results of the vitrectomy technique in anterior segment surgery have been encouraging and in selected cases appear to be an improvement on conventional methods.

Lensectomy has several advantages over conventional intracapsular cataract extraction. The small limbal incision, which frequently does not require to be sutured, allows early ambulation and discharge of the patient from hospital. It is now becoming apparent, however, that with present-day suturing methods this is also possible with standard intracapsular extraction (Galin et al., 1974). The amount of surgically induced astigmatism is small, and patients can be fitted with their final correction earlier. The incidence of vitreous loss is negligible. Should vitreous herniate into the anterior chamber it can readily be removed with the vitrectomy instrument. This is particularly useful in the removal of subluxated lenses. If the lens is too hard for lensectomy then it is possible to do a preliminary anterior vitrectomy, enlarge the corneal incision, and then remove the lens with the cryoprobe.

Lensectomy also has some advantages over phacoemulsification. It is a cheaper and easier technique, which does not require a deep anterior chamber or a large pupil. The posterior capsule can be removed during the initial operation and a subsequent capsulotomy is not required. The main limitation of lensectomy is that it is not, as yet, a suitable technique for the removal of hard cataracts. Although it is possible to remove posterior subcapsular cataracts in elderly patients, who do not have significant nuclear sclerosis, this usually necessitates prolonged manipulation of lens material in the anterior chamber, which increases the risks of endothelial damage and subsequent corneal oedema. Nearly all our severe complications (corneal oedema in 5 eyes and loss of the lens nucleus into the vitreous cavity in 2 eyes) were the direct result of underestimating the hardness of the lens. It is hoped that with more experience with this technique the incidence of these complications will be reduced.

For soft uncomplicated cataracts in children lensectomy is a very good alternative to aspiration. It offers the additional advantage of not requiring a separate infusion system. It also enables a more controlled procedure to be carried out with a more complete removal of cortical lens matter and capsule.

Lensectomy has a significant advantage over aspiration in the management of cataracts secondary to chronic anterior uveitis. These eyes invariably have small pupils with extensive posterior synechiae (Fig. 8a), making access to the lens very difficult. With the vitrectomy instrument the pupil can be enlarged and all adhesions can be freed prior to lensectomy (Fig. 8b). Another advantage of lensectomy is for the removal of cataracts in patients between the ages of 30 and 40 years, who are too 


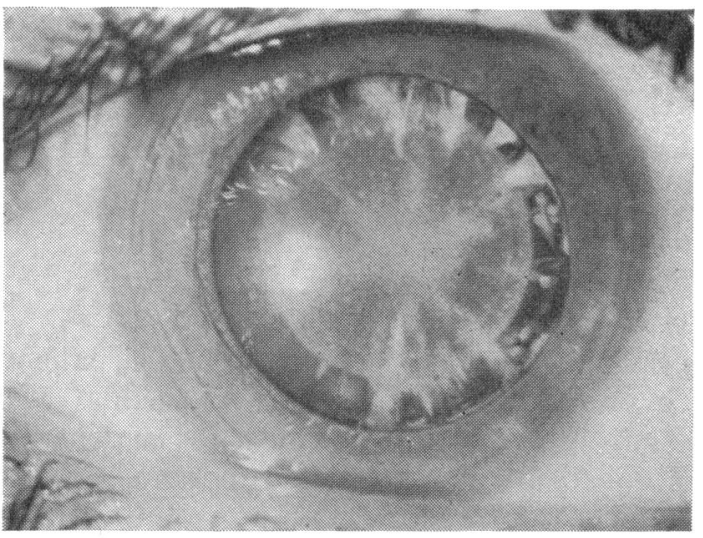

a

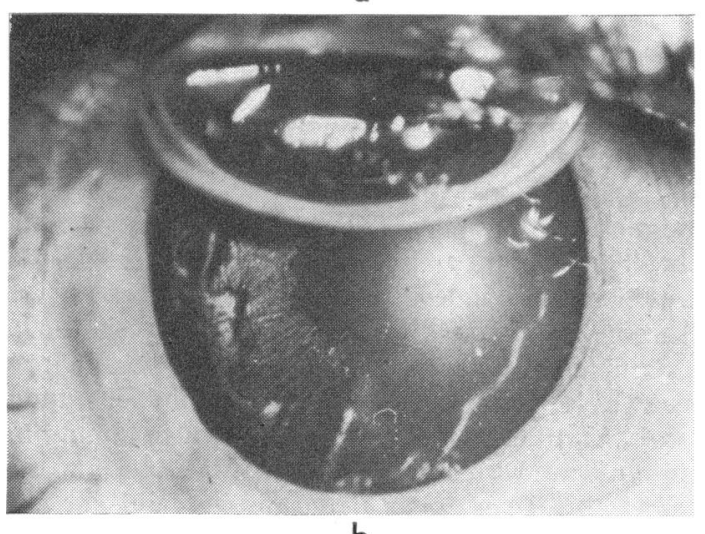

b

Fig. 9 (a) Preoperative appearance of cataract in 30-year-old patient. (b) Postoperative appearance. The posterior capsule has been left intact old for the nucleus to be aspirated but have a high risk of vitreous loss with conventional intracapsular extraction (Fig. 9a, b).

The vitrectomy technique is very useful in anterior segment reconstruction. Some have advocated a pars plana approach (Peyman and Swartz, 1975) to these problems. We feel that, although the pars plana approach may be used in certain cases, we generally prefer to introduce the instrument through a limbal incision so as to avoid the possibility of inducing retinal dialyses. Should a tough membrane be treated with the two-instrument technique then a pars plana approach may be indicated so as to spare the endothelium from prolonged exposure to the infusion stream.

We acknowledge the help and encouragement of $\mathrm{Mr}$ J. R. Hudson, and the surgeons and house surgeons of Moorfields Eye Hospital, High Holborn.

\section{References}

Douvas, N. G. (1973). Transactions of the American Academy of Ophthalmology and Otolaryngology, 77, op. 792.

Federman, J. L., et al. (1974). Presented at a meeting of the Retina Society, Montreal, Canada, September 1974.

Galin, M. A., et al. (1974). Transactions of the American Academy of Ophthalmology and Otolaryngology, 78, 43.

Kloti, R. (1973). Albrecht v. Graefes Archiv fiir Ophthalmologie, 187, 161.

Machemer, R., et al. (1971). Transactions of the American Academy of Ophthalmology and Otolaryngology, 75, 813.

O'Malley, C., and Heintz, R. M. (1972). Transactions of the Pacific Coast Oto-Ophthalmological Society, 53, 121.

Peyman, G. A., and Dodich, N. A. (1971). Archives of Ophthalmology, 86, 548.

Peyman, G. A., and Swartz, M. (1975). Alhrecht v. Graefes Archiv fïr Ophthalmologie, 195, 155. 